Bull. Korean Math. Soc. 51 (2014), No. 5, pp. 1551-1559

http://dx.doi.org/10.4134/BKMS.2014.51.5.1551

\title{
COEFFICIENT DISCS AND GENERALIZED CENTRAL FUNCTIONS FOR THE CLASS OF CONCAVE SCHLICHT FUNCTIONS
}

\author{
BAPPADITYA BHOWMIK AND KARL-JOACHIM WiRTHS
}

\begin{abstract}
We consider functions that map the open unit disc conformally onto the complement of an unbounded convex set with opening angle $\pi \alpha, \alpha \in(1,2]$, at infinity. We derive the exact interval for the variability of the real Taylor coefficients of these functions and we prove that the corresponding complex Taylor coefficients of such functions are contained in certain discs lying in the right half plane. In addition, we also determine generalized central functions for the aforesaid class of functions.
\end{abstract}

\section{Introduction}

Let $\mathcal{A}$ be the class of functions $f$ holomorphic in the unit disc $\mathbb{D}=\{z:|z|<$ $1\}$, where they have a Taylor expansion at the origin of the following form

$$
f(z)=z+\sum_{n=2}^{\infty} a_{n}(f) z^{n}, \quad z \in \mathbb{D} .
$$

We define $C o(\alpha), \alpha \in(1,2]$, as the family of functions $f \in \mathcal{A}$ such that they satisfy the following conditions:

(i) the functions $f \in C o(\alpha)$ are univalent in $\mathbb{D}$,

(ii) the set $\mathbb{C} \backslash f(\mathbb{D})$ is convex and the opening angle of $f(\mathbb{D})$ at infinity does not exceed $\pi \alpha, \alpha \in(1,2]$,

(iii) $f(1)=\infty$.

We call such functions concave univalent functions with opening angle $\pi \alpha$ at infinity and we refer to the articles $[1,2,4,5]$ for a detailed discussion on functions in this class. We now recall the following characterization for functions in $C o(\alpha)$ (compare [2, Theorem 2]):

Received September 4, 2013; Revised April 7, 2014

2010 Mathematics Subject Classification. 30C45.

Key words and phrases. concave function with bounded opening angle at infinity, coefficient region, generalized central functions.

The first author of this article would like to thank NBHM, DAE, India (Ref.No.2/48(20)/2012/NBHM(R.P.)/R\&D-II/14916) for its financial support. 
Theorem A. Let $f \in \mathcal{A}$ and $\alpha \in(1,2]$. A function $f$ belongs to the family $C o(\alpha)$ if and only if there exists a function $\varphi: \mathbb{D} \rightarrow \overline{\mathbb{D}}$, holomorphic in $\mathbb{D}$ such that

$$
\frac{d}{d z}\left(\log \left(f^{\prime}(z)(1-z)^{\alpha+1}\right)\right)=-\frac{(\alpha-1) \varphi(z)}{1-z \varphi(z)}, \quad z \in \mathbb{D}
$$

The present article is dedicated to establish the sets of variability of the functionals $a_{n}(f), f \in C o(\alpha)$ for $n \geq 2$. The exact sets of variability of the coefficients $a_{2}(f)$ and $a_{3}(f)$ are given by (compare [2])

$$
\left\{a_{2}(f): f \in C o(\alpha)\right\}=\left\{w:\left|w-\frac{\alpha+1}{2}\right| \leq \frac{\alpha-1}{2}\right\}
$$

and

$$
\left\{a_{3}(f): f \in C o(\alpha)\right\}=\left\{w=\frac{(\alpha+1)(\alpha+2)}{6}+\frac{\alpha^{2}-1}{3}\left(\zeta+\frac{\alpha-2}{2(\alpha+1)} \zeta^{2}\right): \zeta \in \overline{\mathbb{D}}\right\},
$$

respectively. The boundary points of both the above sets are attained by the second and third Taylor coefficients of the functions

$$
f_{\theta}(z)=\frac{1}{\alpha\left(1-e^{i \theta}\right)}\left(\left(\frac{1-e^{i \theta} z}{1-z}\right)^{\alpha}-1\right), \quad \theta \in(0,2 \pi)
$$

and

$$
f_{0}(z)=\frac{z}{1-z} .
$$

The above results enable us to make the following:

Conjecture 1. Let $\alpha \in(1,2]$, and

$$
f_{\theta}^{\prime}(z)=1+\sum_{n=1}^{\infty} d_{n}(\theta) z^{n}
$$

where $f_{\theta}$ is given by (1.3). We conjecture that for $n \geq 2$ the set of variability of the functional $a_{n}(f), f \in C o(\alpha)$, is the closed simply connected set bounded by the simple closed curve

$$
[0,2 \pi] \ni \theta \rightarrow d_{n-1}(\theta) / n .
$$

In the moment, we are only able to prove that for $n \geq 1$ the set $\left\{d_{n}(\theta)\right.$ : $\theta \in[0,2 \pi]\}$ is a simple closed curve. To achieve this aim, we calculate $d_{n}(\theta)$ explicitly using the Cauchy product formula and we get

$$
d_{n}(\theta)=\frac{\prod_{j=1}^{n}(\alpha+j)}{n !}-(\alpha-1) \sum_{k=1}^{n}\left(\frac{\prod_{j=2}^{k}(j-\alpha)}{k !}\right)\left(\frac{\prod_{j=1}^{n-k}(\alpha+j)}{(n-k) !}\right) e^{i \theta k} .
$$

Let

$$
B_{n}(\zeta)=\sum_{k=1}^{\infty} B_{n, k} \zeta^{k}
$$


where

$$
B_{n, k}=\left(\frac{\prod_{j=2}^{k}(j-\alpha)}{k !}\right)\left(\frac{\prod_{j=1}^{n-k}(\alpha+j)}{(n-k) !}\right), \quad 1 \leq k \leq n,
$$

and $B_{n, k}=0$ for $k>n$. We want to prove that $B_{n}$ delivers a conformal map of the unit disc. The proof of this assertion is an application of the Wolff-Noshiro Lemma which states that $B_{n}$ is univalent on the unit disc if $\operatorname{Re}\left(B_{n}^{\prime}(\zeta)\right)>0$ for $|\zeta|<1$. The inequality $\operatorname{Re}\left(B_{n}^{\prime}(\zeta)\right)>0$ for $|\zeta|<1$ can be proved using the second assertion in [7, p. 107] due to Rogosinski, where one has to consider the case $A_{n}=2$ for all positive integers $n$ assuring that $\operatorname{Re}\left(B_{n}^{\prime}(\zeta)\right)>\frac{1}{2} B_{n, 1}$ if the sequence $\left\{k B_{n, k}\right\}_{k \geq 1}$ is a non-negative monotonically decreasing convex sequence, i.e.,

$$
k B_{n, k} \geq(k+1) B_{n, k+1},
$$

and

$$
k B_{n, k}-2(k+1) B_{n, k+1}+(k+2) B_{n, k+2} \geq 0
$$

for $k \geq 1$. Elementary calculations show the validity of these assertions.

Now, the fact that $\operatorname{Co}(\alpha) \subseteq C o(2)$ for $\alpha \in(1,2]$ implies that the set of variability of $a_{n}(f), f \in C o(\alpha)$, is contained in the set of variability of $a_{n}(f)$, $f \in C o(2)$. Indeed, from [1], we recall that the following set is the exact set of variability for $a_{n}(f), f \in C o(2)$ :

$$
\left\{a_{n}(f): f \in C o(2)\right\}=\left\{w:\left|w-\frac{n+1}{2}\right| \leq \frac{n-1}{2}\right\} .
$$

Recently in [4, Corollary 2.2], the first author of the present article proved that

$$
\max \left\{\left|a_{n}(f)\right|: f \in C o(\alpha)\right\}=\frac{f_{\pi}^{(n)}(0)}{n !},
$$

where $f_{\pi}$ is given by (1.3) with $\theta=\pi$. We now state the following definitions as we need these for our further discussion.

Definition $1.1\left(T_{\delta}\right.$-neighborhood, compare [10]). Let $f \in \mathcal{A}$ have the expansion (1.1). The $T_{\delta}$-neighborhood of $f$ is defined by the following set of functions

$$
T N_{\delta}(f)=\left\{g(z)=z+\sum_{k=2}^{\infty} b_{n} z^{n} \in \mathcal{A}\left|\sum_{k=2}^{\infty} T_{k}\right| a_{k}-b_{k} \mid \leq \delta\right\},
$$

where $\delta \geq 0$ and $T=\left\{T_{k}\right\}_{k \geq 2}$ is a sequence of non-negative real numbers.

The notion of a neighborhood of univalent functions was first introduced by St. Ruscheweyh in [8]. The $T_{\delta}$-neighborhood of $f$ will be called $\delta$-neighborhood of $f$ whenever $T_{k}=k, k=2,3, \ldots$. We also refer to the article [11] for some more interesting results on the $\delta$-neighborhood of the class of Caratheodory functions. In $[6,2.1]$, we introduced the following definition of a central function. 
Definition 1.2 (Central function). Let $\mathcal{F}$ be a class of functions holomorphic in a neighborhood of the origin having a Taylor series expansion of the form (1.1). A function

$$
f_{c}(z)=z+\sum_{n=2}^{\infty} b_{n} z^{n}
$$

is called a central function for the class $\mathcal{F}$ with respect to $\left\{k_{n}\right\}_{n \geq 2}$ if and only if $f_{c} \in \mathcal{F}$ and there exist constants $k_{n}, n \geq 2$, such that the regions of variability for the functionals $a_{n}, n \geq 2$, on the class $\mathcal{F}$ are given by the inequalities

$$
\left|a_{n}-b_{n}\right| \leq k_{n} .
$$

Next, we introduce the notion of a generalized central function.

Definition 1.3 (Generalized central function). A function

$$
f_{g c}(z)=z+\sum_{n=2}^{\infty} b_{n} z^{n}
$$

is called a generalized central function for the class $\mathcal{F}$ with respect to $\left\{k_{n}\right\}_{n \geq 2}$ if and only if $f_{g c} \in \mathcal{F}$ and the constants $k_{n}, n \geq 2$, are such that for each $n \geq 2$, the set of variability for the functional $a_{n}$ on the class $\mathcal{F}$ is contained in the set

$$
\left|a_{n}-b_{n}\right| \leq k_{n}
$$

We comment here that the generalized central function is not unique and we aim to take the sequence $\left\{k_{n}\right\}$ as small as possible.

In [3], Bednarz and Sokól interpreted the inequalities in (1.5) as the existence of a central function $f_{c}$ for $C o(2)$, where

$$
f_{c}(z)=\frac{1}{2}\left(\frac{1}{(1-z)^{2}}-1\right)=\sum_{n=1}^{\infty}\left(\frac{n+1}{2}\right) z^{n}, \quad z \in \mathbb{D},
$$

such that a certain $T_{\delta}$-neighborhood of $f_{c}$ contains $C o(2)$. The question whether a similar approach is possible to compute the radius $\delta$ of the $T_{\delta}$-neighborhood for a generalized central function of the class $C o(\alpha)$, is the reason for the research presented here. In order to fulfill our aim, we first establish the exact sets of variability of the real Taylor coefficients for functions in $C o(\alpha)$. Next, we show that the corresponding complex Taylor coefficients for functions in $\operatorname{Co}(\alpha)$ are contained in certain discs lying in the right half plane. Lastly, as an application of this result, we obtain generalized central functions for the class $C o(\alpha), \alpha \in(1,2]$, such that a certain $T_{\delta}$-neighborhood of this function contains the class $\operatorname{Co}(\alpha)$. We present all these results in the following Section.

\section{Results}

We start this section with the following result: 
Theorem 1. Let $n \geq 2$ and $\alpha \in(1,2]$. Then

$$
\left\{a_{n}(f): f \in C o(\alpha)\right\} \cap \mathbb{R}=\left[1, \frac{f_{\pi}^{(n)}(0)}{n !}\right],
$$

where $f_{\pi}$ is given by (1.3) with $\theta=\pi$.

Proof. In view of (1.5) and (1.6) we only have to show that any point of the interval stated in the Theorem is the $n$-th Taylor coefficient of a function $f \in$ $\operatorname{Co}(\alpha)$. The choice $\varphi(z)=c, c \in[-1,1]$, in (1.2) of Theorem 1 indicates that the functions

$$
g_{c}(z)=\frac{1}{\alpha(1-c)}\left(\left(\frac{1-c z}{1-z}\right)^{\alpha}-1\right), \quad c \in[-1,1),
$$

and

$$
g_{1}(z)=\frac{z}{1-z}, \quad(\varphi(z)=c=1)
$$

are members of the family $C o(\alpha)$. We note that the $n$-th Taylor coefficients of the functions $g_{1}\left(=f_{0}\right)$ and $g_{-1}\left(=f_{\pi}\right)$ are 1 and $f_{\pi}^{(n)}(0) / n$ !, respectively. Hence, the $n$-th Taylor coefficients of these functions $g_{c}, c \in[-1,1]$, fill the interval stated in the theorem. This completes the proof.

Next, in order to describe the set of variability $\left\{a_{n}(f): f \in C o(\alpha)\right\}$, we are only able to prove the following partial result.

Theorem 2. Let $n \geq 2$ and $\alpha \in(1,2]$. Then the set $\left\{a_{n}(f): f \in C o(\alpha)\right\}$ is contained in the closed disc $D_{c_{n}, r_{n}}$ with center

$$
c_{n}=\frac{\prod_{j=1}^{n-1}(\alpha+j)}{n !},
$$

and the radius

$$
r_{n}=\frac{\alpha-1}{n} \sum_{k=1}^{n-1} \frac{\prod_{j=2}^{k}(j-\alpha)}{k !} \frac{\prod_{j=1}^{n-k-1}(\alpha+j)}{(n-k-1) !}=c_{n}-1 .
$$

There exists no smaller disc with the same center than this one containing the above set of variability.

Proof. The sharpness assertion follows directly from formula (1.4) and the fact that $d_{n}(0)=n+1$. This in particular yields $d_{n-1}(0)=n$, which is equivalent to $r_{n}=c_{n}-1$. For the proof of the asserted inclusion relation, we use the following representation formula for functions in $C o(\alpha)$ proved in [2] which states that for any $f \in C o(\alpha)$ there exists a function $\omega: \mathbb{D} \rightarrow \overline{\mathbb{D}}$ holomorphic in $\mathbb{D}$ such that

$$
f^{\prime}(z)=\frac{(1-z \omega(z))^{\alpha-1}}{(1-z)^{\alpha+1}}
$$


For the calculation of the Taylor expansion of the right side of this representation formula we use the following expansion

$$
\frac{1}{(1-z)^{\alpha+1}}=\sum_{n=0}^{\infty}\left(\frac{\prod_{k=1}^{n}(\alpha+k)}{n !}\right) z^{n}, \quad z \in \mathbb{D}
$$

and let

$$
\omega(z)^{r}=\sum_{k=0}^{\infty} t_{k, r} z^{k}
$$

Now using the Cauchy product formula, we get

$$
(n+1) a_{n+1}(f)=\frac{\prod_{j=1}^{n}(\alpha+j)}{n !}-(\alpha-1) \sum_{r=1}^{n} \frac{\prod_{j=2}^{r}(j-\alpha)}{r !} \sum_{k=0}^{n-r} \frac{\prod_{j=1}^{n-r-k}(\alpha+j)}{(n-r-k) !} t_{k, r} .
$$

Hence our assertion is equivalent to

$$
\left|\sum_{r=1}^{n} \frac{\prod_{j=2}^{r}(j-\alpha)}{r !} \sum_{k=0}^{n-r} \frac{\prod_{j=1}^{n-r-k}(\alpha+j)}{(n-r-k) !} t_{k, r}\right| \leq \sum_{r=1}^{n} \frac{\prod_{j=2}^{r}(j-\alpha)}{r !} \frac{\prod_{j=1}^{n-r}(\alpha+j)}{(n-r) !} .
$$

By use of the triangle inequality we see that it is sufficient to prove the inequalities

$$
\left|\sum_{k=0}^{n-r} \frac{\prod_{j=1}^{n-r-k}(\alpha+j)}{(n-r-k) !} t_{k, r}\right| \leq \frac{\prod_{j=1}^{n-r}(\alpha+j)}{(n-r) !} .
$$

Since $|\omega(z)| \leq 1$ implies $\left|\omega(z)^{r}\right| \leq 1$, we may restrict ourselves to the consideration of

$$
\omega(z)=\sum_{k=0}^{\infty} t_{k} z^{k}
$$

and prove

$$
\left|\sum_{k=0}^{N} \frac{\prod_{j=1}^{N-k}(\alpha+j)}{(N-k) !} t_{k}\right| \leq \frac{\prod_{j=1}^{N}(\alpha+j)}{N !} .
$$

To this end, we first consider the polynomial

$$
Q(z)=\sum_{k=0}^{N}\left(\frac{\prod_{j=1}^{N-k}(\alpha+j)}{\prod_{j=1}^{N}(\alpha+j)} \frac{N !}{(N-k) !}\right) z^{k}=: \sum_{k=0}^{N} q_{k} z^{k} .
$$

We know that the Hadamard product or convolution $f_{1} * f_{2}$ of two functions

$$
f_{i}(z)=\sum_{k=0}^{\infty} a_{k}\left(f_{i}\right) z^{k}, \quad i=1,2
$$

is defined as

$$
\left(f_{1} * f_{2}\right)(z)=\sum_{k=0}^{\infty} a_{k}\left(f_{1}\right) a_{k}\left(f_{2}\right) z^{k}
$$


Hence

$$
(Q * \omega)(1)=\sum_{k=0}^{N}\left(\frac{\prod_{j=1}^{N-k}(\alpha+j)}{\prod_{j=1}^{N}(\alpha+j)} \frac{N !}{(N-k) !}\right) t_{k} .
$$

Therefore, the asserted inequality is equivalent to

$$
|(Q * \omega)(1)| \leq 1
$$

Since $|\omega(z)| \leq 1$ for $z \in \mathbb{D}$, it is sufficient to prove that $Q$ is a bound preserving polynomial. According to the Theorems 2.6 and 3.2 in [9] this is the case if

$$
\operatorname{Re}(Q(z))>\frac{1}{2}, \quad z \in \mathbb{D} \text {. }
$$

Due to the result of Rogosinski in [7, p. 107], which we already used in the introduction, the inequality $(2.2)$ is true if we can show that the sequence $\left(\tilde{q_{k}}\right)_{k \geq 0}$ defined by

$$
\tilde{q_{k}}=\left\{\begin{array}{lll}
q_{k} & \text { for } \quad 0 \leq k \leq N \\
0 & \text { for } \quad k \geq N+1
\end{array}\right.
$$

is a monotonically decreasing convex sequence, i.e.,

$$
\tilde{q_{k}}-\tilde{q_{k+1}} \geq 0
$$

and

$$
\tilde{q_{k}}-2 \tilde{q_{k+1}}+\tilde{q_{k+2}} \geq 0
$$

for $k \in \mathbb{N} \cup\{0\}$. The proof of the validity of these inequalities in our case is a simple exercise in elementary calculations. This completes the proof of Theorem 2.

Now, an application of the above theorem yields the following interesting result.

Theorem 3. A generalized central function for the class $\operatorname{Co}(\alpha), \alpha \in(1,2]$, with respect to $\left\{k_{n}\right\}_{n \geq 2}$ where

$$
k_{n}=\frac{\prod_{k=1}^{n-1}(\alpha+k)}{n !}-1
$$

is given by

$$
f_{g c_{\alpha}}(z)=\frac{1}{\alpha}\left(\frac{1}{(1-z)^{\alpha}}-1\right), \quad z \in \mathbb{D} .
$$

Moreover, for any positive sequence $\left\{\sigma_{n}\right\}_{n \geq 2}$ such that

$$
\delta=\sum_{n=2}^{\infty} \sigma_{n}<\infty
$$


the class $C o(\alpha)$ is contained in the $T_{\delta}$-neighborhood of $f_{g c_{\alpha}}$ whenever the sequence $\left\{T_{n}\right\}_{n \geq 2}$ is given by

$$
T_{n}=\sigma_{n}\left(\frac{\prod_{k=1}^{n-1}(\alpha+k)}{n !}-1\right)^{-1}
$$

Proof. We note that the following series expansion is possible for the function $f_{g c_{\alpha}}(z)$ at the origin

$$
f_{g c_{\alpha}}(z)=\frac{1}{\alpha}\left(\frac{1}{(1-z)^{\alpha}}-1\right)=z+\sum_{n=2}^{\infty}\left(\frac{\prod_{k=1}^{n-1}(\alpha+k)}{n !}\right) z^{n} .
$$

Now since $f_{g c_{\alpha}} \in C o(\alpha)$, the proof of the first part of the theorem is over from Theorem 2. To prove the remaining part of the theorem, we calculate the radius $\delta$ for the $T_{\delta}$-neighborhood as follows:

$$
\sum_{n=2}^{\infty} T_{n}\left(\frac{\prod_{k=1}^{n-1}(\alpha+k)}{n !}-1\right)=\sum_{n=2}^{\infty} \sigma_{n}=\delta
$$

Remark. We note here that the $\left\{k_{n}\right\}_{n \geq 2}$ in Theorem 3 are minimal concerning our generalized central function according to Theorem 2 .

Remark. Bednarz and Sokól computed (see f.i. [3, Theorem 4]) the radius $\delta$ for the $T_{\delta}$-neighborhood of

$$
f_{c}(z)=\frac{1}{2}\left(\frac{1}{(1-z)^{2}}-1\right)
$$

with respect to the sequence $\left\{T_{n}\right\}_{n \geq 2}$ where

$$
T_{n}=\frac{1}{n^{2}(n-1)} \quad \text { as } \quad \frac{1}{2}\left(\frac{\pi^{2}}{6}-1\right) .
$$

We now take a special choice of $\sigma_{n}=\frac{1}{2 n^{2}}$ and compute the radius $\delta$ for the $T_{\delta}$-neighborhood of $f_{g c_{\alpha}}(z)=\frac{1}{\alpha}\left(\frac{1}{(1-z)^{\alpha}}-1\right)$ with respect to the sequence $\left\{T_{n}\right\}_{n \geq 2}$ where

$$
T_{n}=\frac{1}{2 n^{2}}\left(\frac{\prod_{k=1}^{n-1}(\alpha+k)}{n !}-1\right)^{-1}
$$

A straightforward computation gives

$$
\begin{aligned}
\delta & =\sum_{n=2}^{\infty} T_{n}\left(\frac{\prod_{k=1}^{n-1}(\alpha+k)}{n !}-1\right) \\
& =\sum_{n=2}^{\infty} \frac{1}{2 n^{2}}=\frac{1}{2}\left(\frac{\pi^{2}}{6}-1\right) .
\end{aligned}
$$


We observe here that the computed radius $\delta$ for the $T_{\delta}$-neighborhood of $f_{g c_{\alpha}}$, $\alpha \in(1,2]$, is same as the result of Bednarz and Sokól but in our case with bigger $T_{n}$ except in the case $\alpha=2$ where their result is the same as ours.

Acknowledgement. The authors would like to thank St. Ruscheweyh for the idea to accomplish the proof of the inequality (2.1) and the referee for his valuable comments.

\section{References}

[1] F. G. Avkhadiev, Ch. Pommerenke, and K.-J. Wirths, Sharp inequalities for the coefficients of concave schlicht functions, Comment. Math. Helv. 81 (2006), no. 4, 801-807.

[2] F. G. Avkhadiev and K.-J. Wirths, Concave schlicht functions with bounded opening angle at infinity, Lobachevskii J. Math. 17 (2005), 3-10.

[3] U. Bednarz and J. Sokól, On T-neighborhoods of analytic functions, J. Math. Appl. 32 (2010), 25-32.

[4] B. Bhowmik, On concave univalent functions, Math. Nachr. 285 (2012), no. 5-6, 606612 .

[5] B. Bhowmik, S. Ponnusamy, and K.-J. Wirths, Characterization and the pre-Schwarzian norm estimate for concave univalent functions, Monatsh. Math. 161 (2010), no. 1, 5975.

[6] B. Bhowmik and K.-J. Wirths, Central functions for classes of concave univalent functions, Math. Slovaca, To Appear.

[7] W. Rogosinski, Über positive harmonische Entwicklungen und typisch reelle Potenzreihen, Math. Z. 35 (1932), no. 1, 93-121.

[8] St. Ruscheweyh, Neighborhoods of univalent functions, Proc. Amer. Math. Soc. 81 (1981), no. 4, 521-527.

[9] T. Sheil-Small, On the convolution of analytic functions, J. Reine Angew. Math. 258 (1973), 137-152.

[10] T. Sheil-Small and E. M. Silvia, Neighborhoods of analytic functions, J. Analyse Math. 52 (1989), 210-240.

[11] J. Sokól, On neighborhoods of analytic functions with positive real part, Math. Nachr. 284 (2011), no. 11-12, 1547-1553.

BAPPADITYA BHOWMIK

DEPARTMENT OF MATHEMATICS

Indian Institute of TECHNOLOGy KhaRAGPUR

KHARAGPUR-721302, INDIA

E-mail address: bappaditya@maths.iitkgp.ernet.in

KARL-JOACHIM WIRTHS

Institut für AnAlysis And Algebra

TU Braunschweig, 38106 Braunschweig, Germany

E-mail address: kjwirths@tu-bs.de 